\title{
凝胶结合剂超细金刚石磨粒工具的制备及应用*
}

\author{
陆 静 罗求发 宋运运 胡光球 徐西鹏 \\ (华侨大学脆性材料加工技术教育部工程研究中心 厦门 361021)
}

\begin{abstract}
摘要: 利用溶胶凝胶原理制备超细粒度金刚石磨料工具。提出对超细金刚石磨料进行剪切分散解决磨料在凝胶结合剂中的分 散问题; 选用复合干燥控制化学添加剂改善凝胶结合剂基体的性能; 加入偶联剂提高凝胶结合剂基体对超细金刚石磨粒的把 持能力。研究结果表明, 经剪切分散后的超细金刚石磨料在凝胶结合剂中分布均匀; 复合添加剂成功抑制了凝胶结合剂基体 的收缩并防止了其脱水失效; 偶联剂通过化学键合的作用有效提高了凝胶结合剂基体对超细金刚石磨粒的把持能力, 减少了 磨粒的脱落。采用所制备的超细金刚石凝胶结合剂工具磨抛加工两种典型的硬脆性基片材料, 获得了纳米级甚至亚纳米级的 超光滑无损表面。
\end{abstract}

关键词: 凝胶结合剂; 超细金刚石; 磨抛加工; 基片

中图分类号: TG356

\section{Fabrication and Application of Gel-bonded Ultrafine Diamond Abrasive Tools}

\author{
LU Jing LUO Qiufa SONG Yunyun HU Guangqiu XU Xipeng \\ (MOE Engineering Research Center for Brittle Materials Machining, Huaqiao University, Xiamen 361021)
}

\begin{abstract}
Ultrafine diamond abrasive tools have been made by sol-gel technique. Shear dispersing is proposed to resolve the uneven distribution of abrasives in the gel bond. Multiple drying control chemical additives are used to improve the property of the bond matrix. And coupling agent is added to enhance the pullout strength of the abrasives from the bond. The results show that ultrafine diamond abrasives distributed uniformly in the gel bond after the shear dispersion. The modified matrix of the tool with compound additives is successfully restrained from shrinkage and prevented from dehydration failure. And the holding force of the abrasives in the matrix is effectively enlarged by chemical bond after adding the coupling agent, avoiding the loss of the abrasives. When lapping and polishing two kinds of typical hard and brittle substrates with this gel-bonded ultrafine diamond tool, an ultra-smooth and scratch-free surface with nanoscale even sub-nanoscale precision can be obtained.
\end{abstract}

Key words: gel bond; ultrafine diamond; lapping and polishing; substrate

\section{0 前言}

纳米科技时代的来临给生物、医学、信息等各 个领域带来了新的挑战, 具有特殊用途的功能器件 能否在 21 世纪取得更大的发展, 在很大程度上取决 于基片的超精密加工技术水平。相关产品性能要求 的不断提高促使超精密加工精度从微米级提高到了 纳米甚至亚纳米级, 并向其极限精度原子级挑战。 在传统 $\mathrm{Si}$ 晶片的化学机械抛光 (Chemical mechanical polishing, CMP)中, 磨料都是分散于抛光 液中, 一般选用比较软的 $\mathrm{SiO}_{2}$ 作为磨料, 但是由于

\footnotetext{
* 国家自然科学基金一广东联合基金重点(U1034006)、国家自然科学基 金青年基金 (51105149) 和高校博士学科点专项科研基金 (20133501130001)资助项目。20140814 收到初稿, 20141211 收到修改稿
}

第三代基片材料如 $\mathrm{SiC}$ 等的硬度非常高, 如果选用 软磨料则加工效率很低。 $\mathrm{LEE}^{[1-3]}$ 在系统研究不同材 料 CMP 加工机理时认为难反应难去除材料的抛光 主要依赖于机械作用而非化学反应, 进而采用添加 纳米金刚石超硬磨粒的方式提高去除率。虽然纳米 金刚石是硬脆性材料超精密抛光的理想磨料 ${ }^{[4-8]}$, 但 是超细磨料的自团聚和加工过程中的再团聚对表面 质量影响很大，且纳米金刚石的引入不仅增加了 CMP 抛光液的使用成本, 还实际回到了磨料运动轨 迹复杂不可控的游离磨料加工阶段。

为了实现材料的高效去除, 国内外研究尝试采 用固结磨料加工方式, 以高速磨削和电解在线砂轮 修整技术(Electrolytic in-process dressing, ELID)磨 削最为典型。研究表明 ${ }^{[9-12]}$, 对于小面积工件, 随 着磨料粒度的减小，这两种方式均可达到纳米级的 
加工精度。但超细磨料固结工具的制备仍沿用粗粒 度磨具粉体混料和烧结成型的工艺过程, 磨粒的团 聚现象不可避免, 而且加工过程中磨粒的有效切除 用量无法控制, 很难获得大面积高质量的加工表面。 由于磨具在抛光过程中会出现磨损, 还需要及时进 行修整, 否则会影响到工件的面形精度。但由于磨 具表面固结了极耐磨的磨料, 使得磨具的修整变得 十分困难和繁琐。同时, 如果条件控制不当, 还极 易在加工过程中对工件产生硬损伤。

可见开发新型的均匀分散且加工轨迹可控的 超细磨料抛光工具是实现硬脆性基片高效超精密加 工的有效途径。虽然超细磨料很难与粉状结合剂均 匀混合制成传统的烧结工具, 但却能分散在特定溶 液中进行固化以用于磨抛加工。日本学者 NAKAMURA ${ }^{[13]}$ 把含有 $\mathrm{SiO}_{2} 、 \mathrm{Al}_{2} \mathrm{O}_{3}$ 和 $\mathrm{Cr}_{2} \mathrm{O}_{3}$ 超细磨 料的海藻酸钠溶胶滴到 $\mathrm{Ca}^{2+}$ 溶液中形成凝胶球, 再 将这些凝胶球黏结成磨抛片, 利用这种工具可以获 得优良的加工效果, 但是使用寿命短且磨料易脱落, 最终制约了其在实践中的推广。在前期工作中尝试 将 W5 到 W40 粒度的氧化铝或金刚石磨料分散在海 藻酸钠溶胶中再凝胶干燥制成了大面积抛光膜, 加 工硅片和大理石等硬度较低的材料获得了比较理想 的表面质量 ${ }^{[14-18]}$ 。虽然海藻酸钠成膜特性良好, 但 是强度低且容易脱水, 在使用过程中存在膜片破损 和干裂失效的问题 ${ }^{[19]}$, 无法用于加工花岗石等硬度 较高的材料。对于超细的金刚石磨料, 简单混合在 溶胶中很难达到磨粒的均匀分散 ${ }^{[20]}$, 且高分子基材 与金刚石界面能较高, 对磨粒的机械把持力不足, 在加工过程中非常容易脱落 ${ }^{[21]}$, 划伤工件的同时也 降低了工具的寿命。本文在此基础上, 提出对超细 金刚石磨料进行剪切分散的思路解决最小粒径达 $100 \mathrm{~nm}$ 的金刚石磨粒在凝胶结合剂中的分散问题; 选用复合添加剂改善凝胶结合剂基体的性能; 通过 加入偶联剂提高凝胶结合剂基体对超细金刚石磨粒 的把持能力。并采用所制备的超细粒度金刚石工具 磨抛加工两种典型硬脆材料基片, 以评价工具的使 用性能。

\section{1 超细金刚石磨料的剪切分散}

溶胶凝胶磨抛垫的制备是基于溶胶凝胶反应 原理。首先是将海藻酸钠粉末溶解于水中, 然后把 分散好的磨料加入海藻酸钠溶胶中搅拌均匀, 接着 再将其涂覆成型, 并利用钙化反应将磨料固结于凝 胶体中, 最后适当干燥形成磨抛工具。

然而由于超细金刚石的比表面积大、比表面能 高、处于热力学不稳定状态, 所以在介质中分散稳
定性差, 容易发生团聚, 使其在应用过程中受到严 重制约 ${ }^{[22]}$ 。为了避免上述缺陷, 需要对超细金刚石 磨料进行剪切分散, 并研究了其在溶液和工具中的 分散效果。

超细金刚石磨料在水溶液中的分散特性：分别 将 $\mathrm{W} 1$ 及 $100 \mathrm{~nm}$ 的金刚石磨料悬浮液用 IKA T18 剪切分散机进行剪切分散, 剪切分散时间为 $3 \mathrm{~min}$, $\mathrm{W} 1$ 金刚石悬浮液的浓度为 $1 \%, 100 \mathrm{~nm}$ 金刚石悬 浮液的浓度为 $0.1 \%$, 分别观察磨料在 $1 \mathrm{~h} 、 2 \mathrm{~h} 、 3 \mathrm{~h}$ 、 $4 \mathrm{~h} 、 5 \mathrm{~h}$ 的沉降幅度。同时对比没有剪切分散的 W1 及 $100 \mathrm{~nm}$ 的金刚石磨料在 $1 \mathrm{~h} 、 2 \mathrm{~h} 、 3 \mathrm{~h} 、 4 \mathrm{~h} 、 5 \mathrm{~h}$ 的沉降幅度。

由图 1 对比可知, 金刚石粒度越细剪切分散后 在水溶液中的稳定性越好, 且超细粒度的金刚石磨 料进行剪切分散能有效避免团聚。

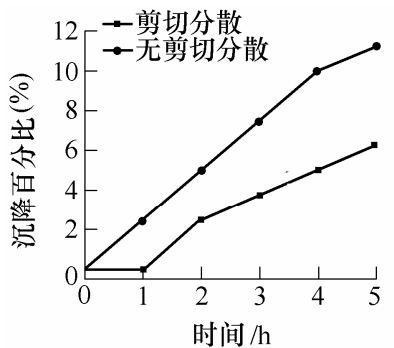

(a) W1

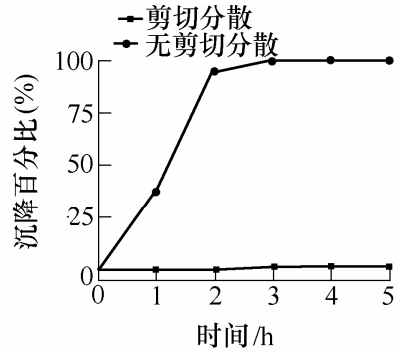

(b) $100 \mathrm{~nm}$
图 1 金刚石磨料的沉降百分比

为了具体量化分析剪切分散后 W1 的金刚石磨 料及 $100 \mathrm{~nm}$ 的金刚石磨料在水溶液中的分散情况, 利用粒度分析仪(Zetasizer nano ZS) 对上述悬浮液进 行粒度分布检测, 并与没有剪切分散的 $\mathrm{W} 1$ 金刚石 及 $100 \mathrm{~nm}$ 金刚石在水溶液中的粒度分布进行对比 试验研究。图 2 为有无剪切分散的 W1 金刚石的粒 度分布图; 图 3 为有无剪切分散的 $100 \mathrm{~nm}$ 金刚石 的粒度分布图。

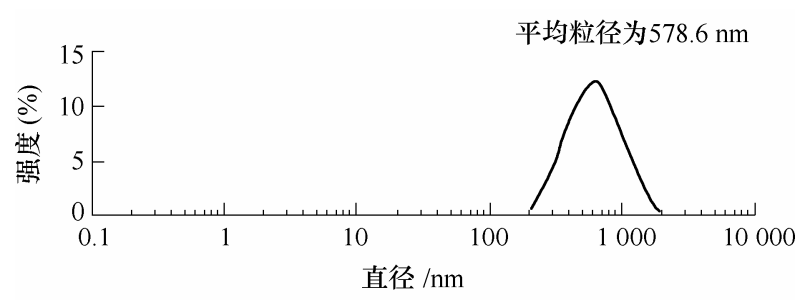

(a) 剪切分散

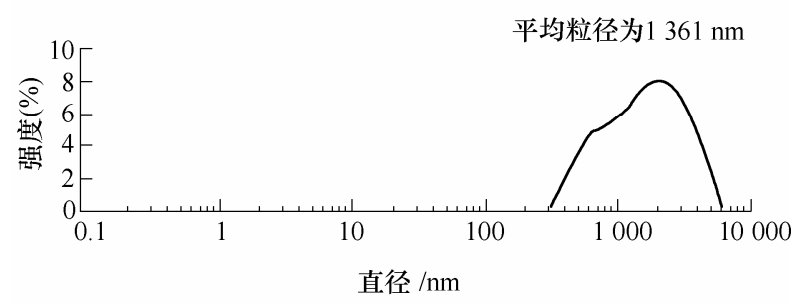

(b) 无剪切分散

图 2 W1 金刚石磨料有无剪切分散的粒度分布 


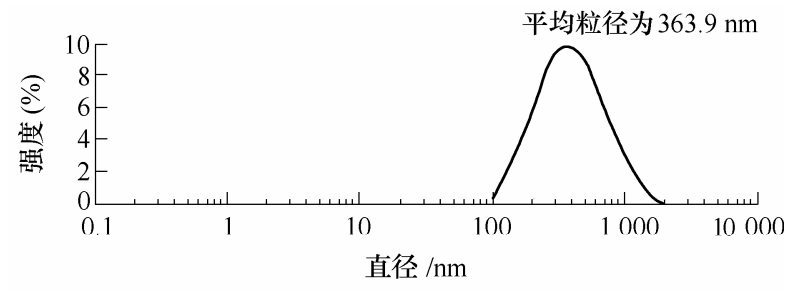

(a) 剪切分散

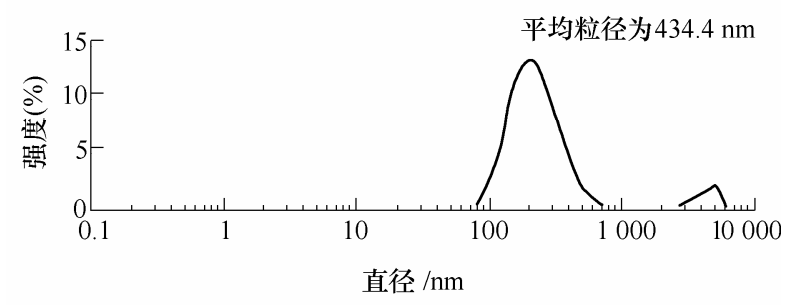

(b) 无剪切分散

图 $3100 \mathrm{~nm}$ 金刚石磨料有无剪切分散的粒度分布

从图 2 中可以看出, 当金刚石磨料粒径为 $\mathrm{W} 1$ 时, 剪切分散后在水溶液中的平均粒径为 $578.6 \mathrm{~nm}$, 如果不进行分散处理, 其粒径达到 $1361 \mathrm{~nm}$, 基本 为两个至三个磨粒团聚在一起, 大于剪切分散后的 平均粒径。而且, 没有剪切分散的悬浮液中团聚比 较严重的磨料在检测过程中已经沉淀, 实际的团聚 体粒径要远大于所测量的数值。从图 3 中可以看出, 当选用的金刚石磨料粒径为 $100 \mathrm{~nm}$ 时, 如果对其 进行剪切分散, 其平均粒径为 $363.9 \mathrm{~nm}$ 。但如果不 进行分散处理, 其粒径达到 $434.4 \mathrm{~nm}$, 大于剪切分 散后的平均粒径, 且存在微米级的团聚体。可以看 出, 剪切处理后磨料的分散效果要明显好于没有剪 切分散的磨料。

超细金刚石磨料在磨抛垫中的均布状态: 将制 备好的磨抛垫(金刚石粒度 $100 \mathrm{~nm}$, 质量分数 $0.1 \%$ ) 用 Phenom 台式扫描电镜观察磨粒的分散情况。图 4 分别是纯机械摚拌和剪切分散后再机械摚拌的磨 料分布图。由图 4 对比可知, 单纯对磨料进行机械 搅拌时磨料极易团聚, 团聚的最大直径达到 $2 \mu \mathrm{m}$ 以上, 分散效果非常差; 而剪切分散后的磨料分布 均匀, 基本没有出现大的团聚体。

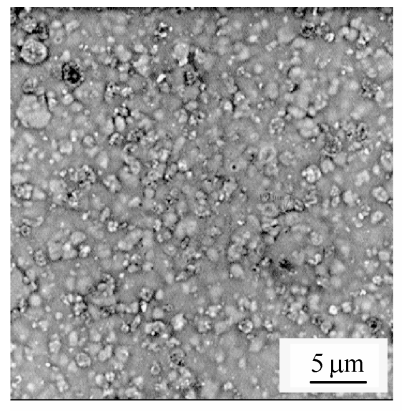

(a) 机械摚拌

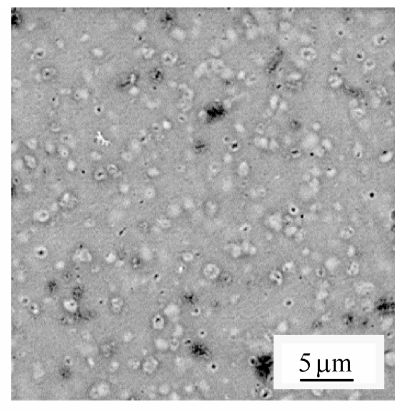

(b) 剪切分散+机械摚拌
图 $4100 \mathrm{~nm}$ 金刚石磨抛垫的 SEM 照片 $(\times 10000)$

\section{2 复合添加剂改性凝胶结合剂基体}

磨抛垫是以海藻酸钠水凝胶作为工具的基体, 制作过程中需要对其进行干燥, 基体收缩过大和持 续脱水又会导致工具的强度低稳定性差。所以通过 添加干燥控制化学添加剂(Drying control chemical additives，DCCA)抑制工具基体的收缩和延长工具 的保存时间。

DCCA 从增强凝胶网络骨架的结构强度和提高 凝胶基体的持水性这两个方面着手，分别选取骨架 填充剂和持水剂两类, 以解决湿凝胶干燥和储存过 程中存在的问题。骨架填充剂选择纳米功能粒子, 由于纳米功能粒子具有大的比表面积和比表面能, 因此具有很高的活性。这类粒子常常用于各类橡胶 的充填, 用来提高橡胶制品的抗老化和力学性能。 持水剂选择含有亲水基团的有机物, 它可以通过减 弱水分蒸发速率, 防止干裂失效, 延长工具的存放 时间和使用寿命。

通过试验探索, 选取 $\mathrm{SiO}_{2}$ 和蔗糖分别作为骨架 填充剂和持水剂, 最优配比为 $2 \%$ 的 $\mathrm{SiO}_{2}(100 \mathrm{~nm})$ 和 10\%的蔗糖。图 5 分别给出了原始体系、二氧化 硅体系、蔗糖体系和复合体系膜片风干 $2 \mathrm{~h}$ 后的效 果图。从图 5 中可以明显看出, 添加二氧化硅和蔗 糖后的复合体系综合了两种单一体系的优点, 体积 收缩和持水能力明显好于原始体系。参照 CMP 中 聚氨酯抛光垫的力学性能指标并结合磨抛垫的基体 特性, 对原始体系和复合体系的体积收缩率、抗拉 强度和硬度进行了检测。表 1 中的结果说明了复合 添加对膜片力学性能影响不大。

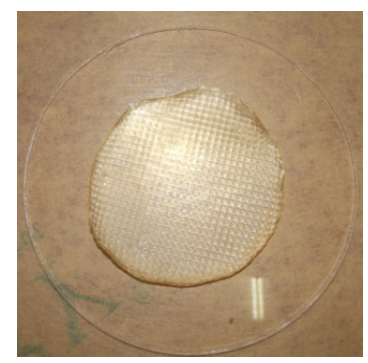

(a) 原始体系

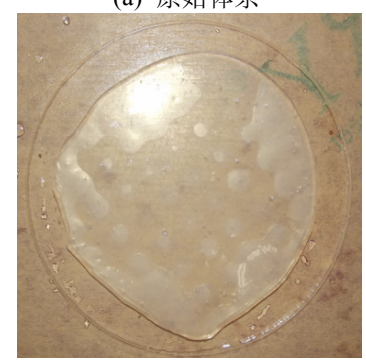

(c) 蔗糖体系

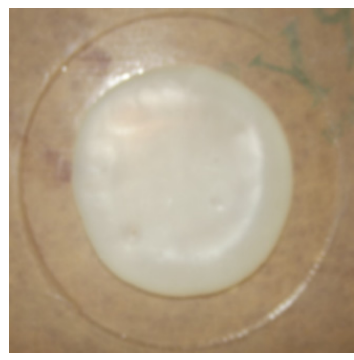

(b) 二氧化硅体系

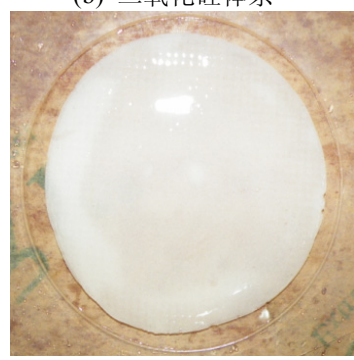

(d) 复合体系
图 5 不同配方制备的圆形膜片风干 $2 \mathrm{~h}$ 后的效果图 
表 1 两种体系膜片的力学指标

\begin{tabular}{ccc}
\hline 力学指标 & 原始体系 & 复合体系 \\
\hline 体积收缩率 $(\%)$ & 90.7 & 73.6 \\
抗拉强度 $/ \mathrm{MPa}$ & 3.1 & 2.9 \\
邵氏硬度 & 40.9 & 38.2 \\
\hline
\end{tabular}

为了观察工具的长期使用和存放稳定性, 图 6 给出了放置五天后工具的表面形貌、截面图和放置 14 天后工具的表面干裂状态。

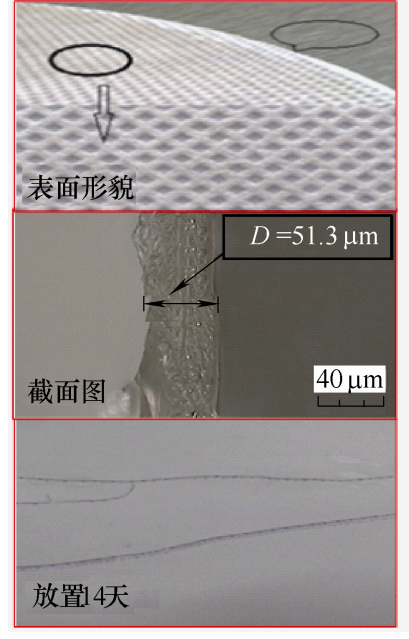

(a) 原始体系

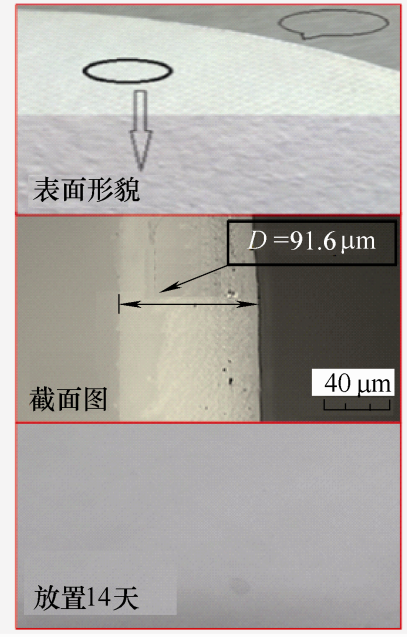

(b) 复合体系
图 6 两种体系制备工具的状态对比

从表面形貌可以看出, 复合体系制备的工具与 原始体系对比，放置 5 天后其边缘没有出现显著的 收缩现象, 这说明 DCCA 能够抑制该工具沿直径方 向的收缩。同时原始体系制备的工具能够清晰的看 到无纺布的网格结构, 而复合体系则看不到, 证实 DCCA 能够抑制该工具沿轴向方向的收缩, 截面图 显示膜层厚度增加了 $78 \%$ 。两种体系的工具在常温 下放置 14 天后, 原始体系制备的工具表面有明显的 干裂, 而复合体系则无裂纹产生。这也说明了复合 添加以后工具的持水性变好稳定性增强。

虽然复合体系抑制了基体的收缩, 提高了基体 的保水能力, 但是同时也导致了基体过于致密, 加 工过程中反而降低了晶片的表面质量。为了克服复 合体系过于致密的缺陷, 往复合体系中添加造孔剂, 它能使水凝胶基体变成一种疏松多孔的结构, 不仅 可以利用造孔的方式来抑制体积的收缩, 而且还能 够增加工具基体的容屑空间。

通过试验探究, 选择十二烷基硫酸钠(Sodium dodecyl sulfate, SDS)作为造孔剂。图 7 给出了在复 合体系的基础上添加不同质量分数 SDS 的工具表 面形貌。随着 SDS 质量分数的增加, 工具表面的孔 也越来越多。当 SDS 质量分数达到 $0.08 \%$ 时, 工具 表面开始出现了泡状结构; 再继续增加 SDS 的质量 分数, 工具表面的孔和泡的数量并无明显增加。这
是因为 $\operatorname{SDS}$ 的临界胶束浓度 (Critical micelle concentration, $\mathrm{CMC}$ ) 为 $8.3 \times 10^{-3} \mathrm{~mol} / \mathrm{L}$, 当 $\mathrm{SDS}$ 质量 分数大于 $0.08 \%$ 时, 海藻酸钠溶胶的表面张力几乎 是一个常数 ${ }^{[23]}$, 因此 SDS 的发泡作用不再发生明显 的变化。综合工具的强度要求, 选定 SDS 的质量分 数为 $0.08 \%$, 在复合体系的基础上形成 SDS 体系。

图 8 给出了单晶硅片三种体系工具加工后的三 维形貌图, 表 2 为单晶硅片三种体系加工后的粗糙 度。加工单晶硅片选用粒度为 $20 \mu \mathrm{m}$ 的氧化铝磨料, 加工参数见表 3 。由对比可知, 经 SDS 体系工具加 工后, 晶片表面比原始体系和复合体系工具加工后 的更光滑、缺陷较少, 且粗䊁度值也更小。

综上, SDS 体系不仅具有复合体系抑制收缩和 保水的能力, 同时还能保证工具的加工精度。

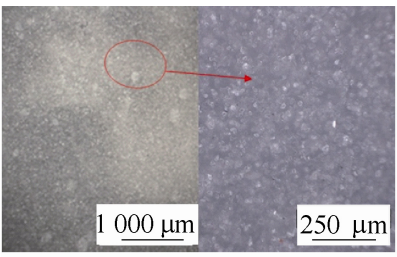

(a) SDS $0 \%$

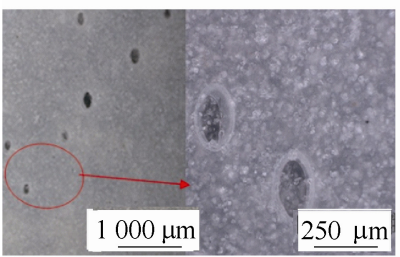

(c) SDS $0.04 \%$

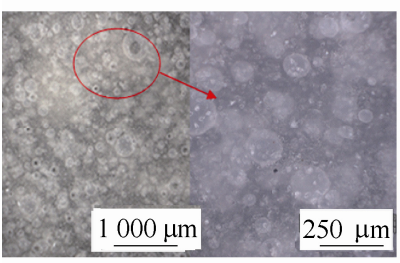

(e) SDS $0.08 \%$

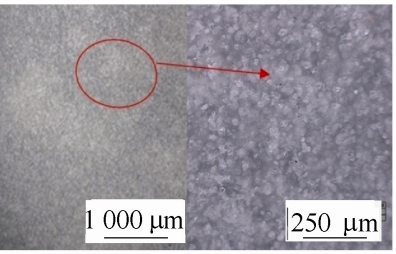

(b) SDS $0.02 \%$

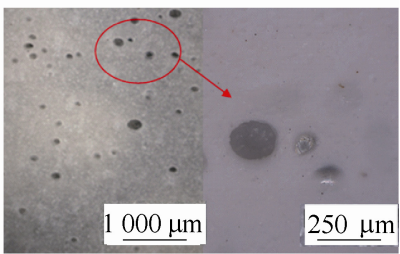

(d) SDS $0.06 \%$

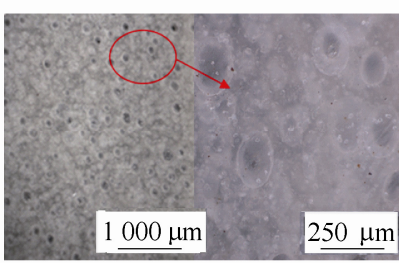

(f) $\operatorname{SDS} 0.10 \%$
图 7 添加不同浓度 SDS 的工具表面形貌

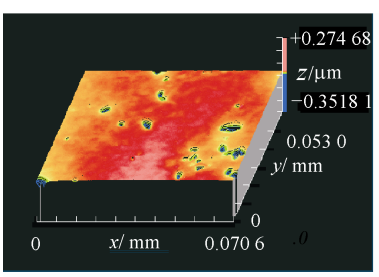

(a) 原始体系

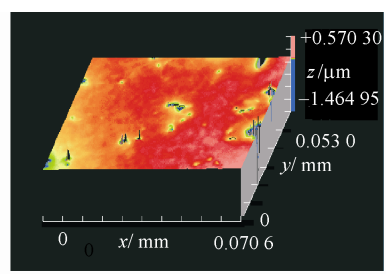

(b) 复合体系

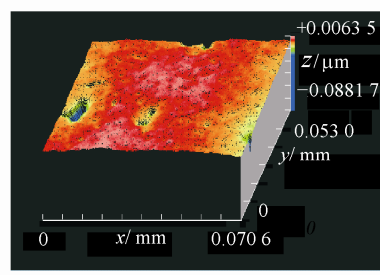

(c) SDS 体系

图 8 单晶硅片三种体系工具加工后的三维形貌 
表 2 单晶硅片三种体系工具加工后的表面粗䊁度

\begin{tabular}{cccccc}
\hline 工具 & 原始体系 & 复合体系 & SDS 体系 \\
\hline 表面粗粘度 $/ \mathrm{nm}$ & 4.2 & \multicolumn{2}{c}{6.4} & 2.1 \\
\hline \multicolumn{6}{c}{} \\
& 表 3 & 单晶硅片的加工参数 \\
\hline 氧化铝粒 & 研磨盘转 & 载样盘转 & 抛光压 & 抛光时 & 抛光液 \\
度 $/ \mu \mathrm{m}$ & 速 $/ \mathrm{r} / \mathrm{min})$ & 速 $/(\mathrm{r} / \mathrm{min})$ & 力 $/ \mathrm{kg}$ & 间 $/ \mathrm{h}$ & 类型 \\
\hline $\mathrm{W} 20$ & 90 & 80 & 2 & 1 & 去离子水 \\
\hline
\end{tabular}

\section{3 偶联剂提高基体对磨料的把持能力}

工具的使用性能除了受磨料和基体本身的影 响外, 还取决于磨料和基体的界面结合强度。由于 金刚石不能为海藻酸钠高分子基材浸润和黏结, 仅 靠机械力的作用部分包裹在结合剂里, 在使用过程 中很容易脱落、流失。尤其是粗抛过程中, 由于磨 料尺寸较大, 其在磨抛垫中的把持力更弱, 加工一 段时间后, 上层磨料脱落严重, 而下层磨料又难以 及时出露, 严重降低了工具的加工效率和使用寿命。 为了增强磨抛垫中基体对磨料的把持力, 通过添加 偶联剂, 使磨料与海藻酸钠产生偶联效果, 提高把 持能力, 减少磨料的脱落。

试验中选用的硅烷偶联剂是 KH550, 用量 $0.1 \%$ 。偶联剂与溶胶体中水分子发生水解反应, 经 缩合后形成低聚结构 ${ }^{[24]}$, 这种低聚结构与磨粒表面 的羟基形成氢键而后共价结合, 有机基团与海藻酸 钠的羧基发生缩合, 最终达到桥连磨粒和基体的作 用, 化学反应过程如图 9 所示。
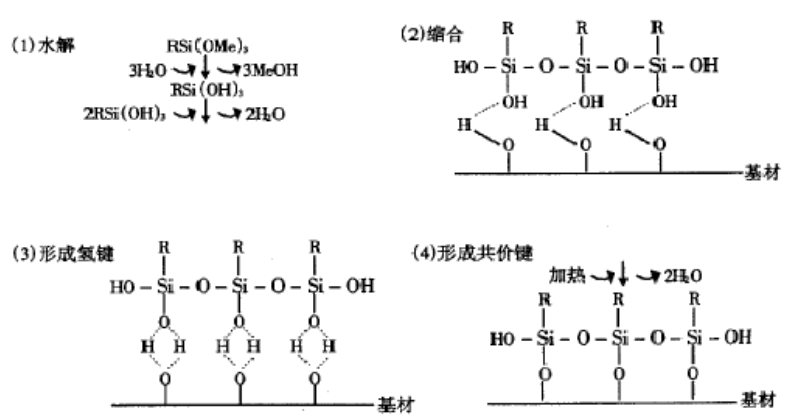

图 9 硅烷偶联剂的化学反应过程

由图 10 可以明显看出, 添加偶联剂改性前后, 磨抛垫中的磨料脱落情况差别很大。未添加偶联剂 的磨抛垫在加工 $60 \mathrm{~min}$ 后, 表层的多数磨料已被磨 平或脱落, 而下层的磨料又还未出露; 添加偶联剂 改性后的磨抛垫中只有少数磨料出现脱落的情况。

图中磨抛垫泛黄是由于磨料脱落而显出海藻酸钠的 颜色。试验表明, 添加偶联剂 KH550 后, 磨料与海 藻酸钠发生了化学键合, 磨抛垫中基体对磨料的把

持力增强, 磨料的脱落情况大为改善。

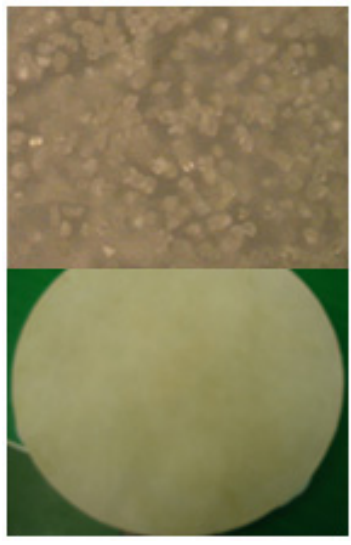

$0 \mathrm{~min}$

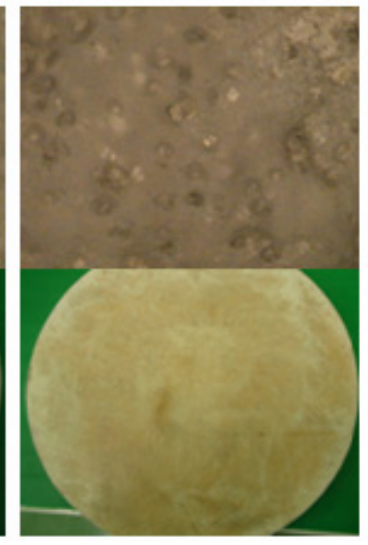

$60 \mathrm{~min}$

(a) 未添加偶联剂

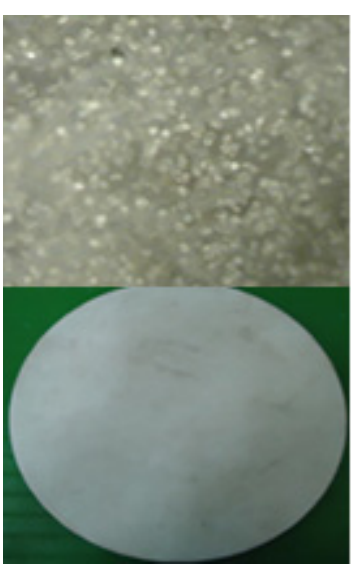

$0 \mathrm{~min}$

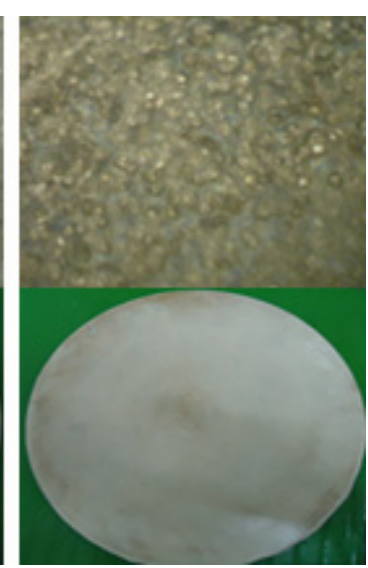

$60 \mathrm{~min}$ (b) 添加偶联剂

图 10 磨抛垫中金刚石磨料的脱落情况 $(\times 500)$

\section{4 加工硬脆材料基片的效果}

改性制备的磨抛垫在硬脆材料加工方面的应 用前景十分广阔, 目前主要用于磨抛碳化硅、陶瓷、 蓝宝石等硬脆性基片。试验使用的加工设备是 Engis 超精密研磨抛光机组和 AUTOPOL-1000S 精密研磨 抛光机，检测设备是 ZYGO 三维表面轮廓仪。

图 11 是先后用金刚石粒度为 W10、W1、W0.1 的磨抛垫加工单晶碳化硅晶片的表面三维形貌图, 加工参数见表 4。碳化硅磨抛加工过程中, 先用粒 度为 W10 的金刚石把晶片表面较大的凹坑去除, 余 下的小凹坑经 W1 的金刚石去除后, 晶片表面已经 非常光滑, 此时再用 $100 \mathrm{~nm}$ 的金刚石进行精抛光, 最终就能获得亚纳米级粗粮度的超光滑无损表面, 粗粘度值分别为 $1.48 \mathrm{~nm} 、 0.55 \mathrm{~nm}$ 和 $0.26 \mathrm{~nm}$ 。从 三维形貌图中也可以看出, 加工后表面质量比较理 想, 无划痕, 同时也说明了用凝胶结合剂磨抛工具 
加工晶片不会产生机械损伤。

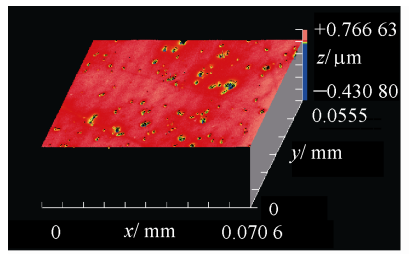

(a) W10

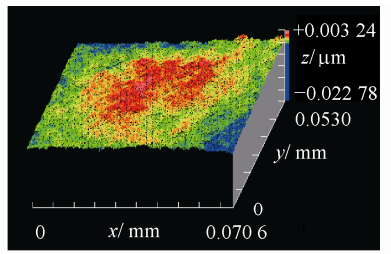

(b) W1

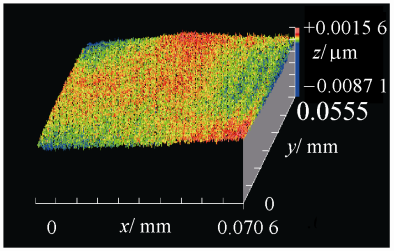

(c) W0.1
图 $11 \mathrm{SiC}$ 晶片三种粒度金刚石磨料加工后的三维形貌

表 4 碳化硅晶片的加工参数

\begin{tabular}{lccccc}
\hline $\begin{array}{c}\text { 金刚石 } \\
\text { 粒度 } / \mu \mathrm{m}\end{array}$ & $\begin{array}{c}\text { 研磨盘转 } \\
\text { 速/ } / \mathrm{r} / \mathrm{min})\end{array}$ & $\begin{array}{c}\text { 载样盘转 } \\
\text { 速 } /(\mathrm{r} / \mathrm{min})\end{array}$ & $\begin{array}{c}\text { 抛光压 } \\
\text { 力 } / \mathrm{kg}\end{array}$ & $\begin{array}{c}\text { 抛光时 } \\
\text { 间 } / \mathrm{min}\end{array}$ & $\begin{array}{c}\text { 抛光液 } \\
\text { 类型 }\end{array}$ \\
\hline $\mathrm{W} 10$ & 90 & 80 & 2.5 & 120 & 去离子水 \\
$\mathrm{W} 1$ & 90 & 80 & 2.5 & 60 & 去离子水 \\
$\mathrm{W} 0.1$ & 90 & 80 & 2.5 & 30 & 去离子水 \\
\hline
\end{tabular}

图 12 是高纯氧化铝基片(纯度 99\%)磨抛前后的 三维形貌图, 表 5 是对应的加工参数。高纯氧化铝 陶瓷是高硬度、高致密度、高耐磨性的硬脆性材料, 加工难度大。且因上一道加工工序使基片表面生成 了大量的凹坑, 而残留下来较大的加工余量, 用粒 度为 W40 的金刚石加工难以去除表面缺陷。所以为 了提高基片的材料去除率和获得理想的抛光表面, 对高硬度的脆性材料抛光前有必要对其进行精密研 磨, 以去除工序余量和表面凹坑。试验证明, 对研 磨后的基片抛光能获得亚纳米级的超光滑无损表 面, 见图 13。
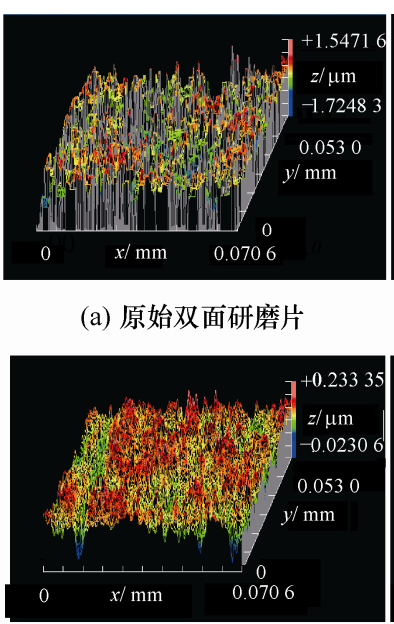

(c) 研磨 $10 \mathrm{~min}$ (a) 原始双面研磨片

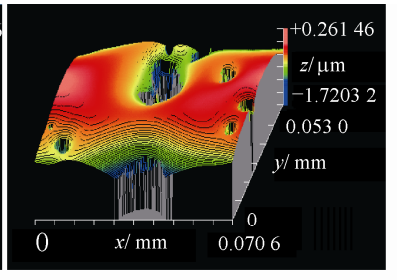

(b) 直接抛光 $4 h(W 40)$

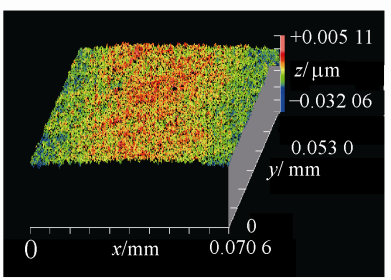

(d) 抛光 $2 h(W 1)$
图 12 氧化铝基片研抛前后的三维形貌
表 5 氧化铝陶瓷基片的加工参数

\begin{tabular}{ccccc}
\hline $\begin{array}{c}\text { 加工 } \\
\text { 类型 }\end{array}$ & $\begin{array}{c}\text { 研磨盘转速 } \\
(\mathrm{r} / \mathrm{min})\end{array}$ & $\begin{array}{c}\text { 载样盘转速 } / \\
(\mathrm{r} / \mathrm{min})\end{array}$ & 抛光压力 & 抛光液类型 \\
\hline 抛光 & 90 & 80 & $2.5 \mathrm{~kg}$ & 去离子水 \\
研磨 & 30 & - & $0.4 \mathrm{MPa}$ & 金刚石研磨液 \\
\hline
\end{tabular}

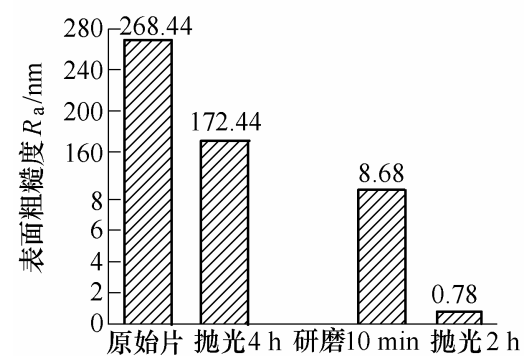

图 13 高纯氧化铝基片研抛加工前后的粗糙度

图 14 为 $\mathrm{SiC}$ 用不同磨料分散方式制备的磨抛 垫精抛后的三维形貌图, 金刚石粒度为 $100 \mathrm{~nm}$, 抛 光时间为 $2 \mathrm{~h}$ 。试验表明对 $\mathrm{SiC}$ 晶片精抛时, 如果不 对超细磨料进行剪切分散而只用单纯的机械摚拌, 晶片表面将产生大量的划痕。这是因为纳米金刚石 极易发生团聚, 团聚体的 “耕犁” 作用直接导致划 痕的产生, 极大的影响了晶片的表面质量, 所以对 纳米金刚石进行剪切分散是非常必要的。两种磨料 分散方式下加工晶片的表面粗粘度分别为 $0.81 \mathrm{~nm}$ 和 $0.26 \mathrm{~nm}$ 。

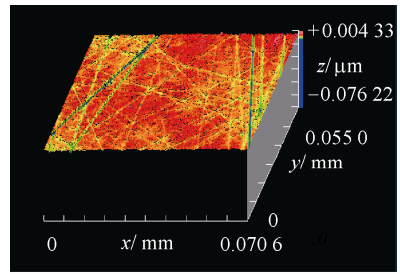

(a) 机械摚拌

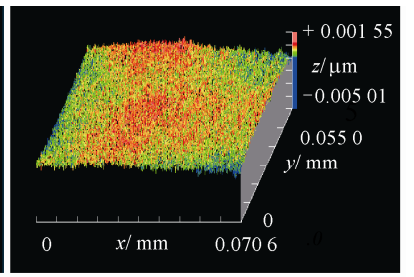

(b) 剪切分散+机械摬拌
图 $14 \mathrm{SiC}$ 相同磨料粒度不同分散方式加工后的三维形貌

\section{5 结论}

（1）超细金刚石磨料在水溶液中经剪切分散后 能有效避免团聚, 稳定性好。在凝胶结合剂中, 经 剪切分散后再机械摚拌的磨粒分布均匀性远好于单 纯的机械搅拌。

(2) 通过添加 DCCA 中的骨架填充剂纳米 $\mathrm{SiO}_{2}$ 和持水剂蔗糖形成复合体系，并在复合体系的基础 上添加造孔剂 SDS, 最终能在不影响工具力学性能 的基础上成功的抑制抛光膜基体的收缩和保证其持 水能力。

(3) 添加偶联剂后, 金刚石磨料与海藻酸钠发 生了偶联效果, 通过化学键合的作用使磨抛垫中基 体对磨料的把持力增强, 磨料的脱落现象大大减少。 
(4) 采用所制备的超细粒度金刚石磨抛垫加工 碳化硅和氧化铝基片, 能够获得纳米级甚至亚纳米 级的超光滑无损表面。

\section{参 考 文 献}

[1] LEE H J, PARK B Y, JEONG S H, et al. The effect of mixed abrasive slurry on CMP of $6 \mathrm{H}-\mathrm{SiC}$ substrates[J]. Journal of Ceramic Processing Research, 2009, 10(3): 378-381.

[2] LEE H S, JEONG H D. Chemical and mechanical balance in polishing of electronic materials for defect-free surfaces[J]. CIRP Annals - Manufacturing Technology, 2009, 58: 485-490.

[3] LEE H S, KIM D I, AN J H, et al. Hybrid polishing mechanism of single crystal $\mathrm{SiC}$ using mixed abrasive slurry (MAS)[J]. CIRP Annals - Manufacturing Technology, 2010, 59: 333-336.

[4] 崔仲鸣, 杨予勇, 王光祖. 纳米金刚石作为抛光材料的 应用[J]. 机械工程与自动化, 2008(4): 198-200.

CUI Zhongming, YANG Yuyong, WANG Guangzu. Discussion on nano-diamond used as polishing material[J]. Mechanical Engineering \& Automation, 2008(4): 198-200.

[5] 王光祖. 纳米金刚石的发展及其应用前景 [J]. 超硬材 料工程, 2008, 20(5): 34-37.

WANG Guangzu. Development and application direction of nanodiamond[J]. Superhard Material Engineering, 2008, 20(5): 34-37.

[6] 胡晓军, 黎明发, 孙振亚, 等. 人造纳米金刚石研究现 状与展望[J]. 武汉理工大学学报, 2009, 31(2): 301-305. HU Xiaojun, LI Mingfa, SUN Zhenya, et al. Research status and prospection of synthetic nanodiamonds[J]. Journal of Wuhan University of Technology, 2009, 31(2): 301-305.

[7] 邹芹, 王明智, 王艳辉. 纳米金刚石的性能与应用前景 [J]. 金刚石与磨料磨具工程, 2003(2): 54-58.

ZOU Qin, WANG Mingzhi, WANG Yanhui. Properties and application prospect of nanometer sized diamond[J]. Diamond \& Abrasives Engineering, 2003(2): 54-58.

[8] KUROBE T, FUJIMURA T, IKEDA H. Nanopolishing of silicon wafers using ultrafine-dispersed diamonds[J]. Physics of the Solid State, 2004, 46 (4): 751-754.

[9] 张飞虎, 朱波, 榇殿荣, 等. ELID 磨削一硬脆材料精 密和超精密加工的新技术[J]. 宇航材料工艺, 1999(1): 51-55.

ZHANG Feihu, ZHU Bo, LUAN Dianrong, et al. ELID grinding - a new technology for precision and ultraprecision machining of hard and brittle materials[J]. Aerospace Materials \& Technology, 1999(1): 51-55.

[10] LIM H S, FATHIMA K, KUMAR A S, et al. A fundamental study on the mechanism of electrolytic in-process dressing (ELID) grinding[J]. International Journal of Machine Tools \& Manufacture, 2002, (42): 935-943.

[11] OHMORI H, NAGAKAWA T. Mirror surface grinding of silicon wafers with electrolytic in process dressing[J], Annals of CIRP, 1990, 39(1): 321-332.

[12] OHMORI H, NAGAKAWA T. Analysis of mirror surface generation of hard and brittle materials by ELID (electrolytic in-process dressing) grinding with superfine grain metallic bond wheels[J]. Annals of CIRP, 1995, 44(1): $287-290$.

[13] NAKAMURA H. Development of a polishing disc containing granulated fine abrasive[J]. Key Engineering Materials, 2003, 238-239: 257-262.

[14] LIU Juan , XU Xipeng. Influences of machining parameters on silicon wafer polished with ultra-fine abrasive polishing pad by gel technique[J]. Key Engineering Materials, 2008, 359-360: 279-284.

[15] LIU Juan, YU Yiqing, XU Xipeng. Fabrication of ultra-fine abrasive polishing pads by gel technique[J]. Materials Science Forum, 2006， 532-533： 468-471.

[16] 匡斌, 徐西鹏. 化学凝胶法制备的超细磨料磨抛工具修 整方法研究 [J]. 金刚石与磨料磨具工程, 2008(2)：5-8. KUANG Bin, XU Xipeng. Study on the dressing methods for gel-based ultra-fine abrasive pads[J]. Diamond \& Abrasive Engineering, 2008(2): 5-8.

[17] LIU Juan, XU Xipeng. Fabrication of ultra-fine diamond abrasive tools by sol-gel[J]. Materials Science Forum, 2004, 471-472: 426-430.

[18] HU Guangqiu, LU Jing, SHEN Jianyun, et al. Surface characterization of silicon wafers polished by three different methods[J]. Key Engineering Materials, 2011, 487: $233-237$

[19] ZHANG Yunhe, LU Jing, HUANG Hui, et al. Effect of fabrication conditions on ultra-fine abrasive polishing pad[J]. Advanced Materials Research, 2012, 565 : 302-306.

[20] HU Guangqiu, LU Jing, XU Xipeng. Polishing silicon wafers with the nanodiamond abrasive tools prepared by sol-gel technique[J]. Key Engineering Materials, 2012, 496: $1-6$.

[21] 徐西鹏, 刘娟, 于怡青, 等. 凝胶结合剂磨粒工具制备 
及其磨抛性能研究 $[J]$. 机械工程学报, 2013，49(19): 156-162.

XU Xipeng, LIU Juan, YU Yiqing, et al. Fabrication and application of gel-bonded abrasive tools for grinding and polishing tools $[\mathrm{J}]$. Journal of Mechanical Engineering, 2013, 49(19): 156-162.

[22] ŌSAWA E J. Recent progress and perspectives in single-digit nanodiamond[J]. Diamond \& Related Materials, 2007, 16: 2018-2022.

[23] KAUSHA D, RANA D S, CHAUHAN M S, et al. The effect of sodium dodecyl sulphate on Furosemide - A cardiovascular drug in water-methanol at different temperature[J]. Journal of Molecular Liquids, 2013, 188: 237-244.

[24] DEY M, DEITZEL J M, GILLESPIE J W, et al. Influence of sizing formulations on glass/epoxy interphase
properties[J]. Composites Part A - Applied Science and Manufacturing, 2014, 63: 59-67.

作者简介: 陆静, 女, 1981 年出生, 博士, 副教授, 硕士研究生导师。 主要研究方向为半导体基片的超精密加工及超细磨料的表面改性。

E-mail: lujing26@hqu.edu.cn

罗求发, 男, 1989 年出生, 博士研究生。主要研究方向为碳化硅晶片和 蓝宝石晶片的超精密加工。

宋运运, 男, 1988 年出生。主要研究方向为磨抛工具的制备和力学性能 评价。

胡光球, 男, 1986 年出生。主要研究方向为超细磨料的分散和碳化硅晶 片的加工。

徐西鹏(通信作者), 男, 1965 年出生, 博士, 教授, 博士研究生导师。 主要研究方向为硬脆材料先进加工技术、超硬材料工具技术、加工过程 监控以及加工过程摩擦学。

E-mail: xpxu@hqu.edu.cn 